\title{
Flavanones common to citrus fruits activate the interferon- stimulated response element by stimulating expression of IRF7
}

\author{
David J. Fast $\mathrm{t}^{*}$, Nathan P. Stern ${ }^{\mathrm{a}}$, Jennifer Chuang ${ }^{\mathrm{b}}$, Yingqin $\mathrm{Li}^{\mathrm{b}}$, \\ Jeffrey D. Scholten ${ }^{\mathrm{a}}$ and Chun $\mathrm{Hu}^{\mathrm{b}}$
}

\author{
aAmway Corporation, 7575 Fulton St. E., Ada, MI 49355, USA \\ bNutrilite Health Institute, 5600 Beach Boulevard, Buena Park, CA 90621, USA \\ ${ }^{*}$ Corresponding author: David J. Fast, Amway Corporation, 7575 Fulton St. E., Ada, MI 49355, USA. Tel: 1-616-787-8604; \\ E-mail: David.Fast@amway.com
}

DOI: $10.31665 /$ JFB. 2019.8207

Received: November 20, 2019; Revised received \& accepted: December 30, 2019

Abbreviations: ACN, acetonitrile; IRF, interferon regulatory factor; ISRE, interferon stimulated response element; IFN $\alpha$, interferon-alpha; $\mathrm{MeOH}$, methanol; MOA, mechanism of action; NFkB, nuclear factor kappa-light-chain-enhancer of activated B cells; OPA, orhto-phosphoric acid; RLU, relative luciferase units

Citation: Fast, D.J., Stern, N.P., Chuang, J., Li, Y., Scholten, J.D., and Hu, C. (2019). Flavanones common to citrus fruits activate the interferon-stimulated response element by stimulating expression of IRF7. J. Food Bioact. 8: 58-65.

\begin{abstract}
Citrus fruits are a rich source of vitamin C and phytochemicals and can be an important part of a healthy diet. Citrus is believed to prevent the occurrence or shorten the duration of symptoms of the common cold and influenza, but meta-analysis of vitamin C clinical trial data is inconclusive. We examined whether citrus flavonoids activated antiviral pathways that might explain the perceived efficacy against the common cold and influenza. We found that a citrus bioflavonoid blend augmented NFKB activation in the presence of imiquimod. In addition, the citrus bioflavonoid blend, as well as individual flavonoids found in the blend, activated the interferon-stimulated response element (ISRE). The ability to activate the ISRE appeared to due to the flavonoids' ability to upregulate expression of the transcription factor interferon regulatory factor 7 (IRF7). Our results suggest that flavonoids from citrus may stimulate antiviral pathways due to their ability to activate the ISRE.
\end{abstract}

Keywords: Antiviral; Citrus; Hesperetin; Interferon; Naringenin; IRF7.

\section{Introduction}

Citrus fruits are a rich source of flavonoids, particularly from their peel and seeds (Tripoli et al., 2007). Flavanones present in citrus include aglycones hesperetin and naringenin. Other classes of flavonoids identified in citrus include flavones (apigenin, luteolin, diosmetin, quercetin, kaempferol) and their respective glycosides; flavanone neohesperidoside (naringin, neohesperidin, poncirin, neoeriocitrin), flavanone rutinoside (narirutin, hesperidin, didymin, eriocitrin, diosmin), and polymethylated flavones (scutellarein, sinensetin, tangeretin, hexamethoxylflavone, nobiletin, and heptamethoxyflavone) (Tripoli et al., 2007).
As a group, flavonoids are strongly implicated as having an important effect on human health (Cassidy et al., 2012; Croft, 1998; Havsteen, 2002). For example, the glycosides hesperidin and naringin are mainly responsible for the purported antioxidant activity of citrus peel extracts in vitro (Kanaze et al., 2009). Likewise, supplementation with hesperidin may affect antioxidant status in animals undergoing physiological challenges. In rats, hesperidin (200 $\mathrm{mg} / \mathrm{kg} /$ day) for 10 days significantly increased levels of superoxide dismutase (SOD), glutathione- $S$-transferase (GST), and GSH after benzo[ $\alpha]$ pyrene-induced oxidative stress (Arafa et al., 2009), while the same dosage of hesperidin did not attenuate antioxidant concentrations in healthy rats. Naringin increased levels of SOD, CAT, and 
vitamin $\mathrm{E}$ in New Zealand White rabbits fed a high cholesterol diet compared to those without the naringin $(0.5 \mathrm{~g} / \mathrm{kg}$ diet $)$ supplement (Jeon et al., 2002). Perche et al found that orange juice or hesperidin, its major flavanone glycoside, did not induce modulation of cellular immune function in healthy well-nourished human subjects (Perche et al., 2014), but antioxidant markers and vascular function were improved in mild hypercholesterolemia subjects when drinking orange juice (Constans et al., 2015) . Therefore, supplementation of flavanone glycosides found in citrus peel extract may be beneficial only when the body is under severe oxidative stress, and when supplementation is given over time and not as a single administration. In addition to potential antioxidant benefits, citrus flavonoids such as narirutin and hesperidin have been reported in rodent models to have hyporglycemic, hypolipedmic, antihypetenisive, antihrombotic, hepatoprotective, and weight management activities (Alam et al., 2014; Hugel et al., 2016; Liu et al., 2008).

Citrus flavonoids may function through multiple mechanisms of action. Interaction with MAPK, PI3 kinase/Akt, NFkB, and PKC signaling pathways may explain their effects on inflammatory markers such as TNF- $\alpha$, IL-6, and MCP-1 (Choi and Ahn, 2008; Choi and Lee, 2010; Vafeiadou et al., 2009). For example, naringenin exhibits anti-inflammatory activity through inhibiting iNOS expression by attenuating p38MAPK and STAT-1 phosphorylation/activation (Vafeiadou et al., 2009). In addition, the effects of flavonoids on different aspects of metabolism may be due to their ability to up-regulate energy regulators AMPK, PPARs, and PGC-1 $\alpha$ (Alam et al., 2014).

Citrus, in particular orange juice, has popularly been used for self-medication for treatment of the common cold and flu. The basis for this folklore is unknown but may due in part to the popularization of vitamin C by Linus Pauling. Multiple clinical studies have been done to evaluate the ability of vitamin $\mathrm{C}$ to prevent or treat cold/flu, and meta-analysis of these studies suggests that vitamin C probably does not prevent infection by rhinoviruses or influenza viruses, but may shorten the duration of disease (Hemilä and Chalker, 2013). Citrus bioflavonoids such as those mentioned above may facilitate vitamin $\mathrm{C}$ absorption (Vinson and Bose, 1988). In Vinson's work on vitamin C absorption, citrus extract contained $19.0 \%$ naringin, $0.34 \%$ naringenin and $0.17 \%$ hesperidin, and promotion of vitamin $\mathrm{C}$ absorption was demonstrated with a 2-gram citrus extract dose containing $500 \mathrm{mg}$ ascorbic acid per oral dose with $380 \mathrm{mg}$ naringin, $6.8 \mathrm{mg}$ naringenin and 3.4 $\mathrm{mg}$ hesperidin. Commercially available citrus bioflavonoid blends include various combinations of citrus fruit extracts. The citrus bioflavonoid blend used as the starting point for this study were standardized to $5.7-6.2 \%$ flavonoids, with profile of three primary flavonoids naringin: narirutin: hesperidin at ratio of $50 \%$ : $15 \%$ : $35 \%$ (structures are seen below).

\section{Materials and methods}

\subsection{Citrus bioflavonoid blend}

Citrus bioflavonoid blend is a mixture of extracts of grapefruit (Citrus $\times$ paradisi), lemon (Citrus limon), mandarin orange (Citrus reticulata) and sweet orange (Citrus sinensis) in a ratio of 20:5:5:70 respectively. These citrus extracts were blended and standardized to $5.7-6.2 \%$ flavonoids, with the three primary flavonoids naringin: narirutin: hesperidin at ratio of 50\%: 15\%: 35\% (Figure 1a). Chemical structures for flavonoids of interest are shown in Figure 1b. Citrus flavonoids were analyzed by HPLC using the following method. Samples were prepared for analysis by weighing $250 \mathrm{mg}$ of citrus bioflavonoid blend and adding $10 \mathrm{~mL}$ of $4 / 1$ Methanol/
DMSO. The solution was vortexed briefly to disperse, sonicated for 15 minutes, and then filtered with $0.22 \mu \mathrm{m}$ PVDF prior to analysis on a Waters H-class UPLC system with a PDA detector. The A mobile phases for UPLC analysis was $0.2 \%$ OPA in water, the B mobile phase was $100 \% \mathrm{MeOH}$, and the $\mathrm{C}$ mobile phase was $100 \%$ acetonitrile. A seven $\mu \mathrm{l}$ sample of each extract was injected into a Waters UPLC HSS $100 \mathrm{~mm}$ analytical column. UPLC separation was carried out as shown below in Table 1 .

\subsection{Reagents}

Naringenin, Narirutin, Hesperetin, Hesperidin, and Imiquimod were purchased from MilliporeSigma (St. Louis, MO). A549 cells (CCL-185) and U-2 OS (HTB-96) were purchased from ATCC (Manassas, VA). McCoy's 5A and Ham's F12K media, amphotericin B, and penicillin/streptomycin were purchased from Mediatech (Manassa, VA). OptiPro serum-free media was purchased from ThermoFisher (Waltham, MA). Fetal bovine serum was purchased from HyClone (Logan, UT). Luciferase reagents were purchased from Biotium (Hayward, CA). RNeasy kit, QIA shredder columns, and SYBR green labeled primers for IRF1, IRF3, IRF7, and IRF9 were purchased from Qiagen (Valencia, CA). iScript cDNA synthesis kit and Ssofast Evagreen supermix were purchased from BioRad (Hercules, CA).

\subsection{Luciferase reporter cell line generation}

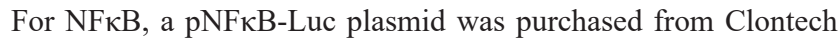
(Mountain View, CA). A 200 bp fragment containing four copies of the NFKB binding consensus sequence and a TATA-like promoter region from the Herpes simplex virus thymidine kinase promoter was excised with NheI and HindIII and gel purified. The fragment was ligated into pGL4.17[luc2/Neo] (Promega, Madison, WI). Plasmid DNA was transfected into human A549 cells using Fugene 6 (Roche, Indianapolis, IN). Cells were incubated in the presence of selective media containing G418 (400 $\mu \mathrm{g} / \mathrm{mL})$ for 10 days and colonies of surviving cells were cloned by limiting dilution. For the ISRE, a pGL4.45[luc2P/ISRE/Hygro] vector (Promega, Madison, WI) was transfected into human osteosarcoma U-2 OS cells using Fugene 6, and cells were incubated in selective media containing hygromycin $(500 \mu \mathrm{g} / \mathrm{mL})$. Resulting clones were tested for optimal responsiveness to IFN $\alpha$.

\subsection{NFkB luciferase reporter assay}

NFkB-luc cells were plated at $4 \times 10^{4} /$ well in white, 96 well, clear bottom microtiter plates and were incubated overnight in OptiPro SFM. The following day, the cells were exposed to samples, imiquimod $(20 \mu \mathrm{M})$, and IL-1 $\beta(50 \mathrm{pg} / \mathrm{mL})$. Six hours after addition of IL-1 $\beta$, the cells were lysed and the relative amount of luciferase was assessed using luciferin reagents from Biotium (Hayward, CA). Data are expressed as per cent control in which the relative luminescence units elicited by control cells exposed only to IL- $1 \beta$ is set at $100 \%$.

\subsection{ISRE luciferase reporter assay}

ISRE-luc cells were plated at $2 \times 10^{4} /$ well in white, 96 well, clear bottom microtiter plates and were incubated overnight in McCoy's $5 \mathrm{~A}-10 \%$ FBS. The following day, cells were exposed to samples. 
a

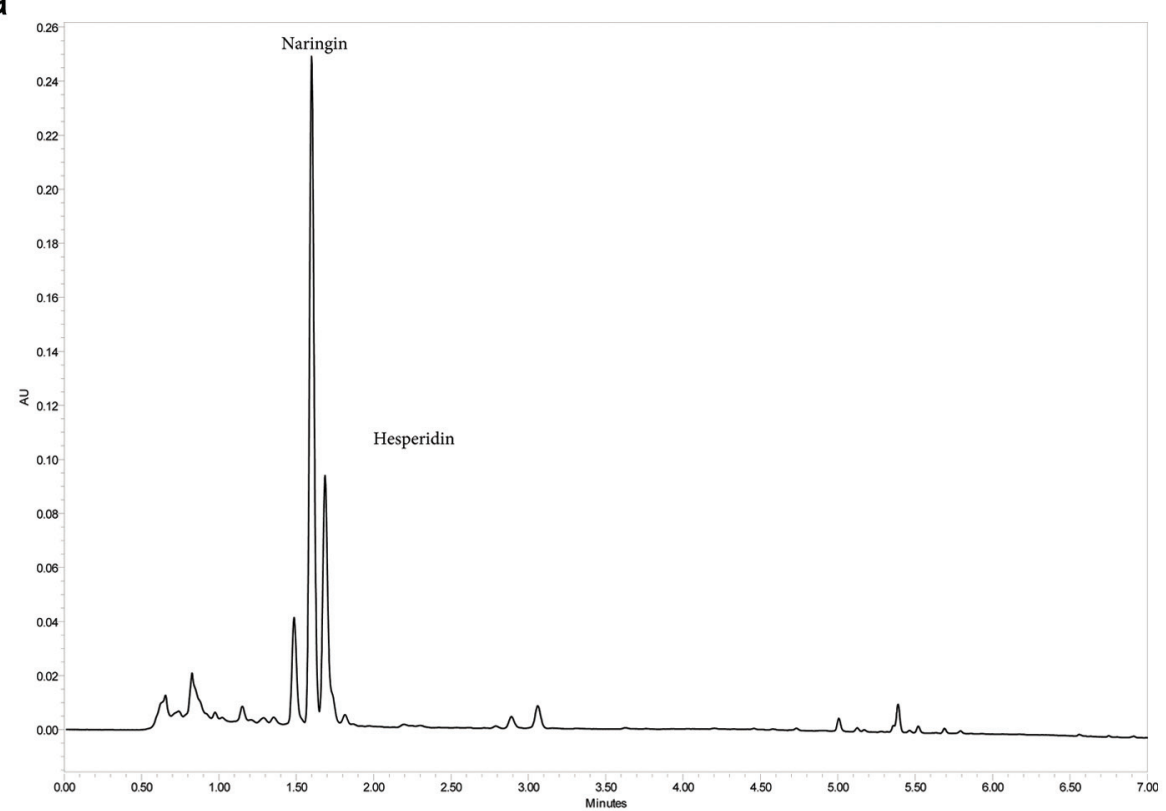

b<smiles>COc1ccc(C2CC(=O)c3c(O)cc(O)cc3O2)cc1O</smiles><smiles>CCOc1cc(O)c2c(c1)OC(c1ccc(OC)c(O)c1)CC2=O</smiles>

Hesperetin

Hesperidin<smiles>O=C1CC(c2ccc(O)cc2)Oc2cc(O)cc(O)c21</smiles>

Naringenin<smiles>CCOc1cc(O)c2c(c1)OC(c1ccc(O)cc1)CC2=O</smiles>

Narirutin

Figure 1. Chemistry of Citrus Bioflavonoid Blend. (a) Representative UHPLC Chromatogram of Proprietary Citrus bioflavonoid blend Extract at $280 \mathrm{~nm}$. Major component peaks identified and labeled as Hesperidin and Naringin. (b) Structures of hesperetin, hesperidin, naringenin and narirutin.

Recombinant human IFNa (PBL Sciences, Tarrytown, NJ) was used as a positive control $(1,000 \mathrm{IU} / \mathrm{mL})$. The cells were incubated overnight, and the relative amount of luciferase activity was assessed the following morning using luciferin reagents from Biotium.

\subsection{Real-time quantitative PCR analysis}

U-2 OS cells were plated in 12 -well plates $\left(5 \times 10^{5}\right.$ cells/well $)$ and grown in McCoy's 5a media supplemented with 10\% FBS and incubated $24 \mathrm{~h}$ in a humidified, $37^{\circ} \mathrm{C}, 5 \% \mathrm{CO}_{2}$ incubator. The following day, flavonoid sample stock solutions were prepared with
DMSO which were then diluted in growth media prior to adding to the cells. The cells were then incubated for an additional 18 h. The media was aspirated, and RNA harvested using Qiashredder columns and an RNeasy kit per the manufacturer's specifications. Total RNA was quantified by A260/A280 ratio, diluted to $1 \mu \mathrm{g}$ per reaction, and reverse transcribed using an iScript cDNA synthesis kit (BioRad). Real-time qPCR reactions were performed using SsoFast EvaGreen qPCR mix on a CFX96 Real-Time Thermocycler (Bio-Rad). The reaction conditions were as follows: 95 ${ }^{\circ} \mathrm{C}$ for $30 \mathrm{sec} ; 45$ cycles of $58{ }^{\circ} \mathrm{C}$ for $5 \mathrm{sec}$; and $95{ }^{\circ} \mathrm{C}$ for $5 \mathrm{sec}$. Fluorescent detection was measured following completion of each cycle. Cycle times of the IRF genes were normalized to $\beta$-actin, a housekeeping gene, prior to comparisons with control samples. 
Table 1. Instrument Conditions

\begin{tabular}{llll}
\hline \multirow{2}{*}{ Time (min.) } & \multicolumn{3}{c}{ Mobile Phase Gradient } \\
\cline { 2 - 4 } & A: 0.2\% OPA & B: MeOH & C:ACN \\
\hline 0 & $60 \%$ & $25 \%$ & $15 \%$ \\
3.2 & $45 \%$ & $35 \%$ & $20 \%$ \\
4.8 & $20 \%$ & $40 \%$ & $40 \%$ \\
5.8 & $10 \%$ & $45 \%$ & $45 \%$ \\
5.81 & $0 \%$ & $50 \%$ & $50 \%$ \\
7 & $0 \%$ & $50 \%$ & $50 \%$ \\
7.01 & $60 \%$ & $25 \%$ & $15 \%$ \\
10 & $60 \%$ & $25 \%$ & $15 \%$ \\
\hline
\end{tabular}

Column: Waters UPLC HSS T3, Length: $100 \mathrm{~mm}$, ID: $2.1 \mathrm{~mm}, 1.8 \mu \mathrm{m}$ particle size; Flow Rate: $0.33 \mathrm{~mL} / \mathrm{min}$; Column Temperature: $25^{\circ} \mathrm{C}$; Sample Vial Temp: $20^{\circ} \mathrm{C}$; Injection Volume: $0.7 \mu \mathrm{L}$; Detection Wavelength: $280 \mathrm{~nm}$; Seal Wash: $90 / 10 \mathrm{v} / \mathrm{v}$ DI Water/ACN; Needle Wash: ACN; Needle Purge: $\mathrm{MeOH}$; Run Time: 10 minutes.

\subsection{Statistical analysis}

The results of most experiments described here are expressed as mean \pm SD values and are representative of three independent experiments. Statistical analysis was carried out by student's t test using PRISM version 7.01 statistical analysis software (GraphPad Software, Inc., San Diego).

\section{Results}

\subsection{Citrus bioflavonoid blend augments imiquimod and IL-16 stimulated activation of NFKB}

While screening botanical extracts for an ability to influence IL$1 \beta$-stimulated activation of an NFKB -linked luciferase reporter, we

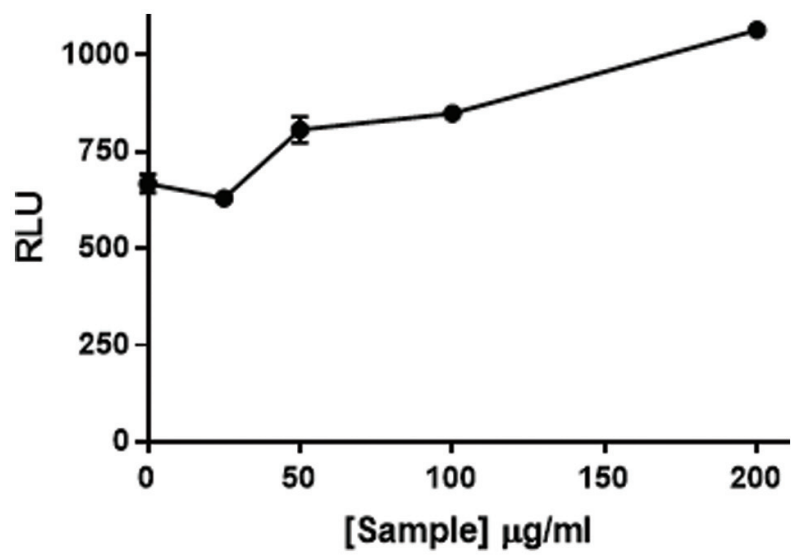

Figure 2. Citrus bioflavonoid blend flavonoid mixture augments NFKB activation. NF49 cells stably transfected with an NFKB response element-luciferase construct were treated with increasing levels of a mixture of citrus flavonoids prior to NFKB activation with imiquimod and human IL-1B (50 $\mathrm{pg} / \mathrm{mL}$ ). The cells were incubated for $6 \mathrm{hr}$. at which time luciferase activity was measured on a Spectramax M5 plate reader. Data from a representative experiment are expressed as RLU compared to positive control cells treated with IMQD and IL-1 $\beta$ only.

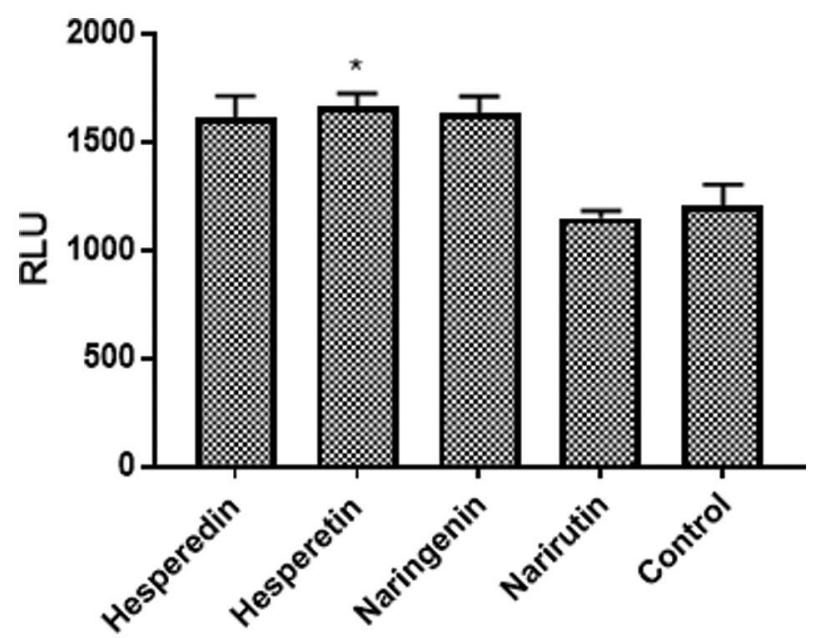

Figure 3. Individual Flavonoids augment NFKB activation. NF49 cells were treated with flavonoids $(20 \mu \mathrm{M})$ know to be present in the citrus mixture prior to NFKB activation with IMQD and human IL-1B (50 pg/mL). The cells were incubated for $6 \mathrm{hr}$. at which time luciferase activity was measured on a Spectramax M5 plate reader. Data from a representative experiment are expressed as RLU compared to positive control cells treated with IMQD and IL-1 $\beta$ only.

observed that several citrus extracts augmented activation of $\mathrm{NF} \kappa \mathrm{B}$ (data not shown). As the TLR7 ligand imiquimod also augments activation of NFKB in our model, we speculated whether citrus flavonoids and imiquimod together might augment activation of $\mathrm{NF \kappa B}$ to a degree greater than either sample alone. The data in Figure 2 demonstrate that in the presence of imiquimod, the citrus bioflavonoid blend did augment activation of $\mathrm{NF} \kappa \mathrm{B}$, in a dose dependent manner, above the degree to which IMQD alone activates NFאB.

To further investigate this observation, we tested several of the individual flavonoids known to be present in the citrus bioflavonoid blend mixture. The data in Figure 3 demonstrate that the response to hesperetin, its glycoside hesperidin, and naringenin all augmented the NFkB response although only the response to hesperetin was significant. Narirutin, the glycoside of naringenin however, had no activity.

\subsection{Citrus bioflavonoids activate the ISRE}

The fact that the citrus bioflavonoid blend augmented NFKB activation by IMQD and IL- $1 \beta$ suggested that compounds found in the citrus bioflavonoid blend might augment anti-viral activity as IMQD is a TLR7 agonist. In addition to activation of NFkB, binding of TLR7 ligands stimulates gene expression of interferon via activation of the ISRE. Therefore, we speculated whether the citrus bioflavonoid blend might also influence activation of a pathway(s) under control of the ISRE. To test this hypothesis, we exposed a stable ISRE-luciferase cell line to the citrus bioflavonoid blend as well as several flavonoids found in the mixture. The data in Figure $4 \mathrm{a}$ demonstrate that the citrus bioflavonoid blend, hesperetin, and naringenin induced expression of luciferase while hesperidin and narirutin did not. One possible mechanism by which flavonoids may activate the ISRE is by inducing production of type I interferon which might act in an autocrine manner. To investigate this possibility, we treated cells with hesperetin, naringenin, and IFN $\alpha$ in the presence or absence of an anti-IFN $\alpha$ antibody. The data in Figure $4 \mathrm{~b}$ show that under the conditions tested, the antibody inhibited 


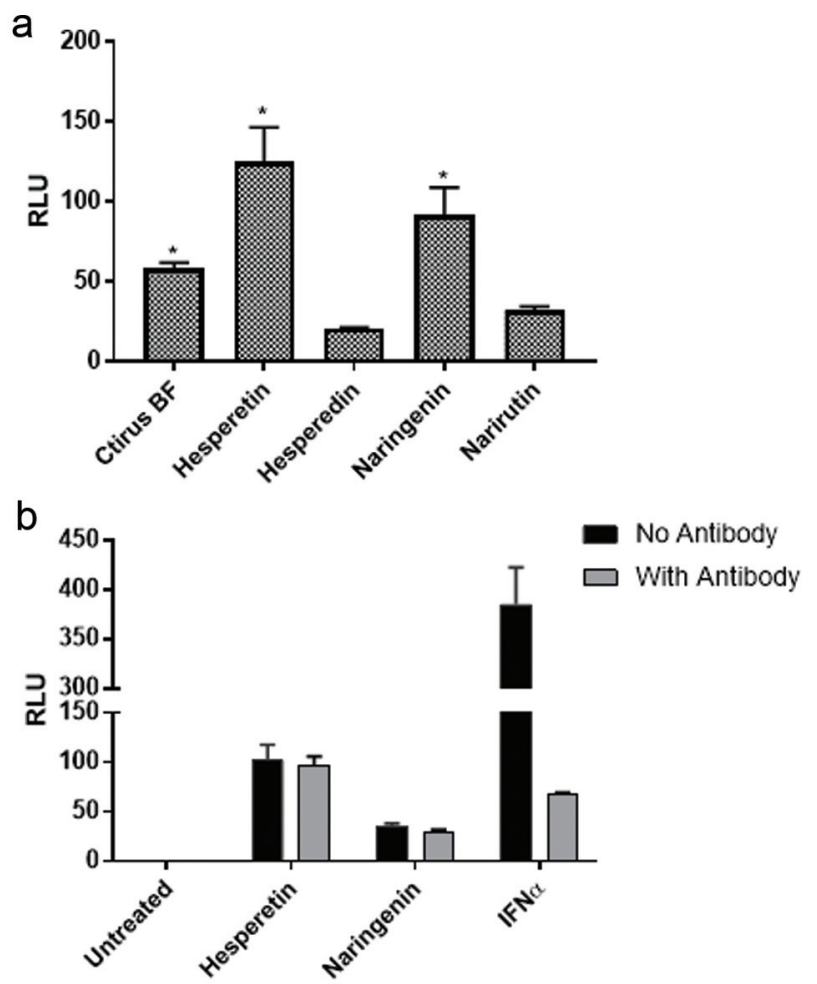

Figure 4. Aglycones but not glycosides activate the ISRE. (a) U2OS cells stably transfected with an ISRE-luciferase plasmid were treated with flavonoids $(20 \mu \mathrm{M})$ known to be present in the citrus mixture. The cells were incubated for $18 \mathrm{hr}$. at which time luciferase activity was measured on a Spectramax M5 plate reader. Data from triplicate wells are expressed as RLU compared to untreated negative control cells. (b) U2OS cells stably transfected with an ISRE-luciferase plasmid were treated with flavonoids $(20 \mu \mathrm{M})$ in the presence or absence of anti-IFN $\alpha$ antibody. Recombinant human universal type I IFN was used as a positive control. Data from triplicate wells are expressed as RLU compared to untreated negative control cells. the response to the IFN $\alpha$ control by approximately $80 \%$ but had little effect on the response induced by the two flavonoids. These results suggest that naringenin and hesperetin activate the ISRE by a mechanism other that simply inducing expression of IFN $\alpha$. As such, the possibility existed that combining naringenin or hesperetin with a low concentration of interferon might activate the ISRE to a level greater than could be stimulated by either alone. The data in Figure 5 demonstrate that naringenin and hesperetin both enhanced the response to a suboptimal concentration of IFN $\alpha$ in a dose dependent manner. At all concentrations of flavonoid tested, the combined response was greater than would be expected if the response was additive. In our hands the response of these cells plateaus when exposed to IFN $\alpha$ at a concentration of 1,000 IU/ $\mathrm{mL}$ (unpublished observation). As the response to flavonoids and low dose IFN $\alpha$ is approximately three-fold higher than the maximum response stimulated by IFN $\alpha$ alone, these results suggest that flavonoids interact with whatever the rate limiting step is in the response of the cells to IFN $\alpha$.

\subsection{Citrus flavonoids induce gene expression of IRF7}

Another such mechanism by which flavonoids might activate the ISRE would be to augment expression of IRFs, the transcription factors that bind the ISRE. To test this possibility, U2OS cells were treated with flavonoids and gene expression of several IRFs was assessed by RT-qPCR. The data in Figure 6a show that expression of IRF7, but not of the other IRFs tested, was upregulated in response to naringenin by 30 minutes with maximal expression by $1 \mathrm{hr}$. The data in Figure 6b show that IRF7 expression increased in a dose dependent manner in cells treated with naringenin for $1 \mathrm{hr}$. Finally, as shown in Figure 6c, only IRF7 expression increased in a dose dependent manner in cells exposed to hesperetin for $1 \mathrm{hr}$

\section{Discussion}

Consumption of an abundance and variety of plant based foods is in general correlated with health benefits (Lampe, 1999), and con-

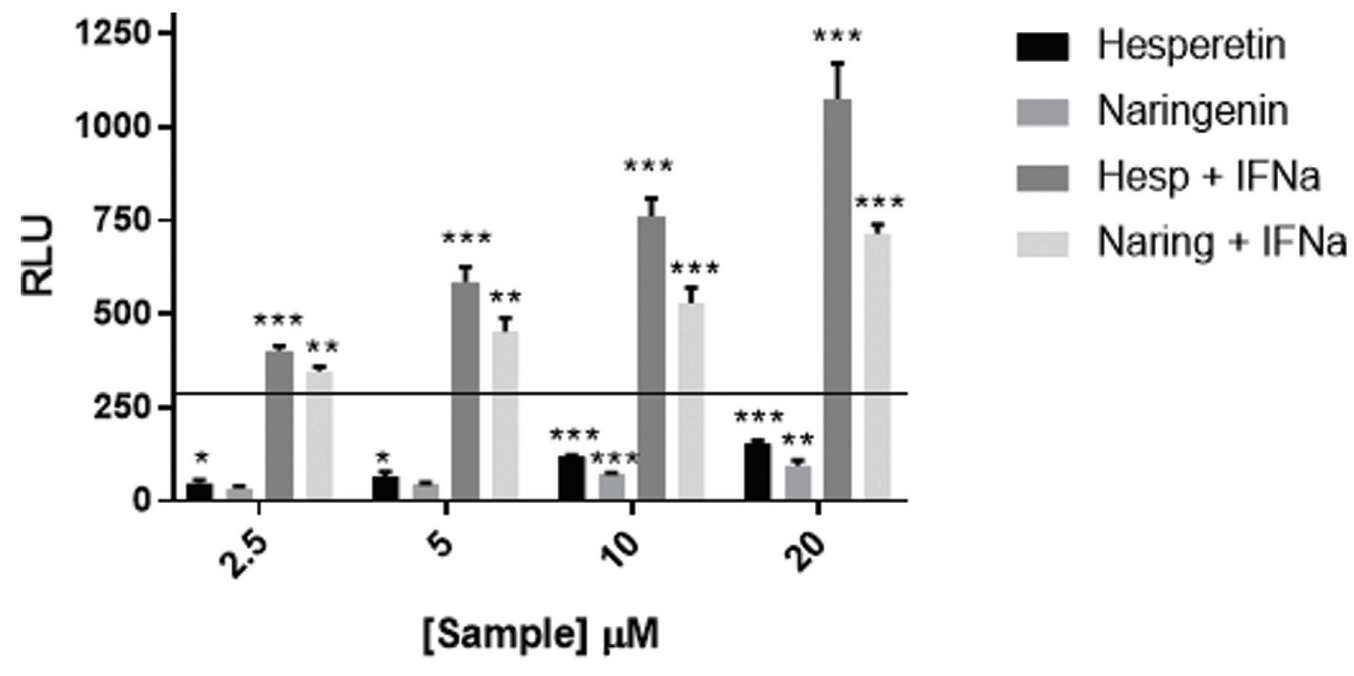

Figure 5. Flavonoids augment ISRE response to IFN $\alpha$. U2OS cells stably transfected with an ISRE-luciferase plasmid were treated with hesperetin and naringenin at the concentrations shown and with IFNa at $200 \mathrm{IU} / \mathrm{mL}$. The cells were incubated for $18 \mathrm{hr}$. at which time luciferase activity was measured on a Spectramax M5 plate reader. Data from triplicate wells are expressed as RLU compared to untreated negative control cells. Recombinant human universal type I IFN was used as a positive control. Data from triplicate wells are expressed as RLU compared to untreated negative control cells. 


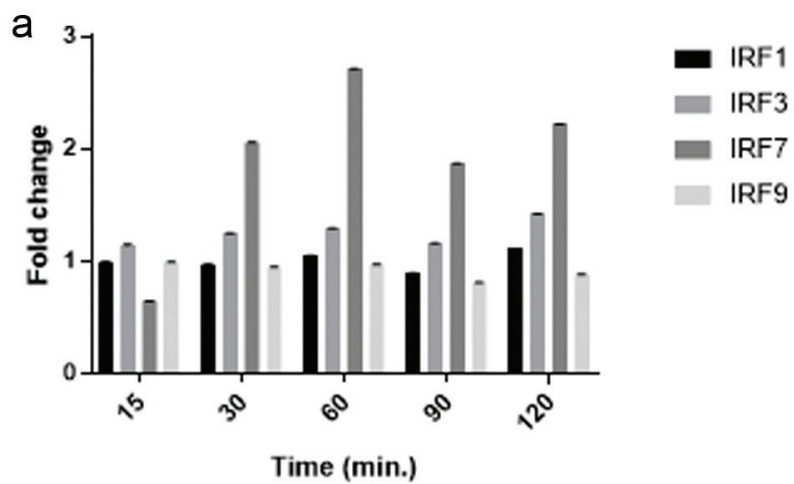

b

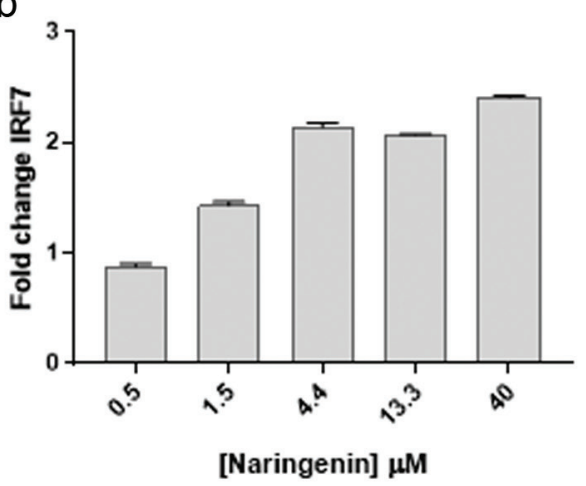

C

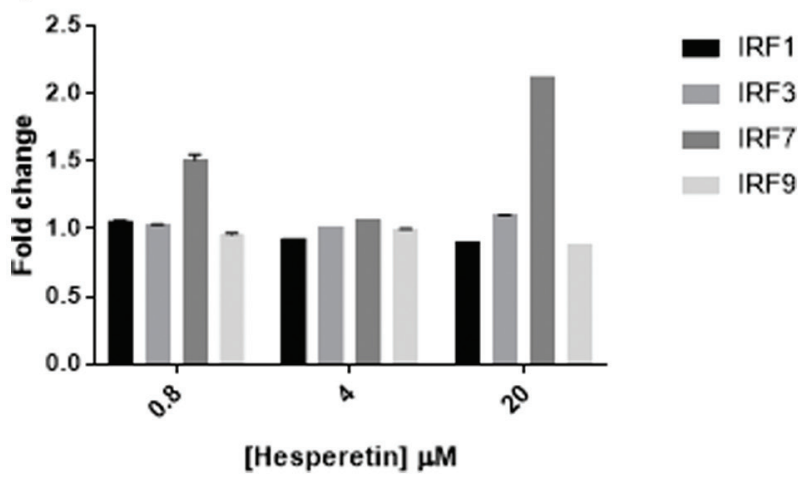

Figure 6. Naringenin and hesperetin stimulate IRF7 gene expression. (a) Time course of IRF expression following treatment of cells with naringenin. U2OS cells were treated with naringenin $(20 \mu \mathrm{M})$ for the lengths of time indicated. RNA was collected using Qiagen RNEasy columns and 2 step PCR was performed using BioRad iScript and Evagreen SsoFast reagents and primers for IRF1, IRF3, IRF7, and IRF9. Data from quadruplicate wells are expressed as mean fold change $\pm S D$ compared to actin as a reference gene. (b) Dose response of IRF7 expression in cells treated with naringenin. U2OS cells were exposed to naringenin at the concentrations indicated for $1 \mathrm{hr}$. and expression of IRF7 was assessed by PCR as described above. Data from quadruplicate wells are expressed as mean fold change \pm SD compared to actin as a reference gene. (c) Dose response of IRF expression in cells treated with hesperetin. U2OS cells were exposed to hesperetin at the concentrations indicated for $1 \mathrm{hr}$. and expression of IRF7 was assessed by PCR as described above. Data from quadruplicate wells are expressed as mean fold change \pm SD compared to actin as a reference gene.

sumption of citrus fruits accounts for a significant portion of overall fruit intake in the majority of geographic diet clusters (Murphy et al., 2014). One of these benefits might be disease prevention through stimulation of antiviral activity as phytochemicals have been shown to impact several antiviral mechanisms (Kapoor et al., 2017; Saha et al., 2009). Direct effects of such phytochemicals include those with the ability to inhibit entry of viruses into cells as well as those with the ability to inhibit viral replication (Kapoor et al., 2017). In addition, phytochemicals can have indirect antiviral effects through stimulation of cellular antiviral mechanisms. These antiviral effects are mediated primarily by receptors of the innate immune system called toll-like receptors (TLR) that recognize viral nucleic acids to stimulate production of inflammatory cytokines and interferons important for elimination of viruses (Kawai and Akira, 2007)

Based on an unpublished observation that several citrus extracts augmented imiquimod and IL-1 $\beta$ stimulated activation of an NFkB-luciferase reporter cell line, the goal of this study was to investigate a potential role of citrus derived flavonoids in the initiation of antiviral activity. In this study, we observed that a citrus bioflavonoid blend augmented IL- $1 \beta$ and imiquimod stimulated activation of NFKB (Figure 2), and that this activity was also found in response to some of the individual flavonoids found in the blend (Figure 3). In contrast to these results, others have found that naringenin and hesperetin had anti-inflammatory effects (Choi and Lee, 2010; Vafeiadou et al., 2009). It is unknown why our results were different from these authors but could be due to different cell types and/or different stimuli (i.e. LPS/IFN $\gamma$ ) stimulating different toll-like receptors.

In addition to augmenting NFkB activation, we observed that the citrus bioflavonoid blend activated an ISRE-luciferase reporter cell line, and that hesperetin and naringenin, but not their respective glycosides hesperidin and narirutin, appear to be responsible for that activity (Figure 4a). Thus, steric hindrance may play a role either in entry of the flavonoids into cells or in binding their molecular target(s). In addition, it appears that hesperetin and naringenin do not act by inducing IFN $\alpha$ production as an antibody to IFN $\alpha$ did not inhibit the response to either hesperetin or naringenin but did abolish the stimulatory effect of recombinant human IFNo on the ISRE reporter (Figure $4 \mathrm{~b}$ ). These results suggested that the citrus flavonoids MOA in the activation of the ISRE is different from the MOA of IFN $\alpha$. To initially investigate this possibility, we tested whether hesperetin and naringenin combined with a suboptimal concentration of IFN $\alpha$ would induce a greater ISRE-luciferase response than either reagent alone. As a matter of fact, the response to hesperetin or naringenin with IFN $\alpha$ was much greater than the response that would be expected due to a simple additive response (Figure 5).

One possible explanation for the apparent synergistic ISRE activation by flavonoids and IFNa is that the flavonoids stimulated expression of an IRF. To initially test this possibility, U2-OS cells were exposed to naringenin for up to $2 \mathrm{hr}$. and expression of IRF1, IRF3, IRF7, and IRF9 was determined by RT-qPCR. Of these, only IRF7 was upregulated by naringenin with maximal expression occurring by $1 \mathrm{hr}$. of treatment. Likewise, the only IRF upregulated by hesperetin was IRF7. These results suggest that hesperetin and naringenin stimulate expression of IRF7. By themselves, this is sufficient to activate the ISRE, and in the presence of IFN $\alpha$ leads to significant activation of the ISRE.

Secondary metabolites from citrus possess a number of biological properties including anti-inflammatory effects (Lv et al., 2015). For example, naringenin has been shown to reduce production of pro-inflammatory cytokines in human blood cells (Bodet et al., 2008) while hesperidin reduced inflammation in a mouse model of LPS-induced lung inflammation (Yeh et al., 2007). This contrasts with what we observed in that hesperetin, hesperidin, and narirutin all augmented activation of $\mathrm{NF \kappa B}$ in a luciferase reporter assay. 
We speculate that the difference between our results and others in the effect of flavonoids on inflammation is due to engagement of different toll-like receptors (TLR) (Medzhitov et al., 1998).The aforementioned studies made use of endotoxin, a TLR4 agonist. In contrast, we used imiquimod, a TLR7 agonist (Hemmi et al., 2002).

Of citrus derived flavonoids, approximately $95 \%$ are flavanones. These exist in both aglycone and glycoside forms in which the aglycones are linked to a sugar moiety (Lv et al., 2015). This is also reflected in our UPLC chromatogram on the citrus bioflavonoid blend in which naringin and hesperidin are the major components. Conversion of the glycoside forms of these flavonoids into their respective aglycone forms undoubtedly occurs in the presence of the gut microbiome (Manach and Donovan, 2004; Nielsen et al., 2006) to release naringenin and hesperetin so that they can act on their molecular targets. Both hesperetin and naringenin were found in plasma after their glycoside forms such as hesperidin and naringin rich citrus juice ingestion by human subject (Erlund et al., 2001; Nielsen et al., 2006). Kaul et al found hesperetin but not the intracellular replication of virus in a monolayer cell (Kaul et al., 1985). In summary, our results suggest that citrus flavonoids stimulate anti-viral activity mediated by upregulation of IRF7 gene expression. To our knowledge, this is a unique antiviral activity of citrus flavonoids.

\section{Conclusion}

The results presented here suggest that aglycone forms of flavanones commonly found in citrus fruits have the potential to activate antiviral pathways under the control of the ISRE and interferon $\alpha$ Specifically, the flavanones tested upregulated expression of IRF7, a transcription factor important in defense against influenza virus infection.

\section{Acknowledgments}

The authors wish to thank Mark Proefke and Christine Hernandez for their support of this work.

\section{Author contributions}

D.F. and C.H. wrote the main manuscript; N.P. performed the UPLC analysis; D.F. performed the cell culture experiments and data analyses. C.H. revised the manuscript. All authors designed the study and reviewed the manuscript.

\section{References}

Alam, M.A., Subhan, N., Rahman, M.M., Uddin, S.J., Reza, H.M., and Sarker, S.D. (2014). Effect of citrus flavonoids, naringin and naringenin, on metabolic syndrome and their mechanisms of action. Adv. Nutr. 5(4): 404-417. doi:10.3945/an.113.005603.

Arafa, H.M., Aly, H.A., Abd-Ellah, M.F., and El-Refaey, H.M. (2009). Hesperidin attenuates benzo[alpha] pyrene-induced testicular toxicity in rats via regulation of oxidant/antioxidant balance. Toxicol. Ind. Health 25(6): 417-427. doi:10.1177/0748233709106624.

Bodet, C., La, V., Epifano, F., and Grenier, D. (2008). Naringenin has antiinflammatory properties in macrophage and ex vivo human wholeblood models. J. Periodontal Res. 43(4): 400-407.

Cassidy, A., Rimm, E.B., O’Reilly, E.J., Logroscino, G., Kay, C., Chiuve, S.E., and Rexrode, K.M. (2012). Dietary flavonoids and risk of stroke in women. Stroke 43(4): 946-951. doi:10.1161/strokeaha.111.637835.

Choi, E.J., and Ahn, W.S. (2008). Neuroprotective effects of chronic hesperetin administration in mice. Arch. Pharm. Res. 31(11): 1457-1462. doi:10.1007/s12272-001-2130-1.

Choi, E.M., and Lee, Y.S. (2010). Effects of hesperetin on the production of inflammatory mediators in IL-1beta treated human synovial cells. Cell Immunol 264(1): 1-3. doi:10.1016/j.cellimm.2010.05.006.

Constans, J., Bennetau-Pelissero, C., Martin, J.F., Rock, E., Mazur, A., Bedel, A., and Berard, A.M. (2015). Marked antioxidant effect of orange juice intake and its phytomicronutrients in a preliminary randomized cross-over trial on mild hypercholesterolemic men. Clin. Nutr. 34(6): 1093-1100. doi:10.1016/j.clnu.2014.12.016.

Croft, K.D. (1998). The Chemistry and Biological Effects of Flavonoids and Phenolic Acids a. Ann. N. Y. Acad. Sci. 854(1): 435-442.

Erlund, I., Meririnne, E., Alfthan, G., and Aro, A. (2001). Plasma kinetics and urinary excretion of the flavanones naringenin and hesperetin in humans after ingestion of orange juice and grapefruit juice. J. Nutr. 131(2): 235-241.

Havsteen, B.H. (2002). The biochemistry and medical significance of the flavonoids. Pharmacol. Ther. 96(2-3): 67-202.

Hemilä, H., and Chalker, E. (2013). Vitamin C for preventing and treating the common cold. Cochrane Database of Systematic Reviews (1): CD000980. doi:10.1002/14651858.CD000980.pub4.

Hemmi, H., Kaisho, T., Takeuchi, O., Sato, S., Sanjo, H., Hoshino, K., and Akira, S. (2002). Small anti-viral compounds activate immune cells via the TLR7 MyD88-dependent signaling pathway. Nat. Immunol. 3(2): 196.

Hugel, H.M., Jackson, N., May, B., Zhang, A.L., and Xue, C.C. (2016). Polyphenol protection and treatment of hypertension. Phytomedicine 23(2): 220-231. doi:10.1016/j.phymed.2015.12.012.

Jeon, S.M., Bok, S.H., Jang, M.K., Kim, Y.H., Nam, K.T., Jeong, T.S., and Choi, M.S. (2002). Comparison of antioxidant effects of naringin and probucol in cholesterol-fed rabbits. Clin. Chim. Acta 317(1-2): 181-190

Kanaze, F.I., Termentzi, A., Gabrieli, C., Niopas, I., Georgarakis, M., and Kokkalou, E. (2009). The phytochemical analysis and antioxidant activity assessment of orange peel (Citrus sinensis) cultivated in Greece-Crete indicates a new commercial source of hesperidin. Biomed. Chromatogr. 23(3): 239-249. doi:10.1002/bmc.1090.

Kapoor, R., Sharma, B., and Kanwar, S. (2017). Antiviral phytochemicals: an overview. Biochem. Physiol. 6(2): 7.

Kaul, T.N., Middleton, E. Jr, and Ogra, P.L. (1985). Antiviral effect of flavonoids on human viruses. J. Med. Virol. 15(1): 71-79.

Kawai, T., and Akira, S. (2007). Antiviral signaling through pattern recognition receptors. J. Biochem. 141(2): 137-145.

Lampe, J.W. (1999). Health effects of vegetables and fruit: assessing mechanisms of action in human experimental studies. Am. J. Clin. Nutr. 70: 475S-490S.

Liu, L., Xu, D.M., and Cheng, Y.Y. (2008). Distinct effects of naringenin and hesperetin on nitric oxide production from endothelial cells. J. Agric. Food Chem. 56(3): 824-829. doi:10.1021/jf0723007.

Lv, X., Zhao, S., Ning, Z., Zeng, H., Shu, Y., Tao, O., and Liu, Y. (2015). Citrus fruits as a treasure trove of active natural metabolites that potentially provide benefits for human health. Chem. Cent. J. 9(1): 68.

Manach, C., and Donovan, J.L. (2004). Pharmacokinetics and metabolism of dietary flavonoids in humans. Free Radical Res. 38(8): 771-786.

Medzhitov, R., Preston-Hurlburt, P., Kopp, E., Stadlen, A., Chen, C., Ghosh, S., and Janeway Jr, C.A. (1998). MyD88 is an adaptor protein in the hToll/IL-1 receptor family signaling pathways. Molecular Cell. 2(2): 253-258.

Murphy, M.M., Barraj, L.M., Spungen, J.H., Herman, D.R., and Randolph, R.K. (2014). Global assessment of select phytonutrient intakes by level of fruit and vegetable consumption. Br. J. Nutr. 112(6): 1004-1018.

Nielsen, I.L.F., Chee, W.S., Poulsen, L., Offord-Cavin, E., Rasmussen, S.E., Frederiksen, H., and Williamson, G. (2006). Bioavailability is improved by enzymatic modification of the citrus flavonoid hesperidin in humans: a randomized, double-blind, crossover trial. J. Nutr. 136(2): 404-408.

Perche, O., Vergnaud-Gauduchon, J., Morand, C., Dubray, C., Mazur, A., and Vasson, M.P. (2014). Orange juice and its major polyphenol hes- 
peridin consumption do not induce immunomodulation in healthy well-nourished humans. Clin. Nutr. 33(1): 130-135. doi:10.1016/j. clnu.2013.03.012.

Saha, R.K., Takahashi, T., and Suzuki, T. (2009). Glucosyl hesperidin prevents influenza a virus replication in vitro by inhibition of viral sialidase. Biol. Pharm. Bull. 32(7): 1188-1192.

Tripoli, E., Guardia, M.L., Giammanco, S., Majo, D.D., and Giammanco, M. (2007). Citrus flavonoids: Molecular structure, biological activity and nutritional properties: A review. Food Chem. 104(2): 466-479. doi:10.1016/j.foodchem.2006.11.054.
Vafeiadou, K., Vauzour, D., Lee, H.Y., Rodriguez-Mateos, A., Williams, R.J., and Spencer, J.P. (2009). The citrus flavanone naringenin inhibits inflammatory signalling in glial cells and protects against neuroinflammatory injury. Arch. Biochem. Biophys. 484(1): 100-109. doi:10.1016/j.abb.2009.01.016.

Vinson, J.A., and Bose, P. (1988). Comparative Bioavailability to Humans of Ascorbic Acid Alone or in Citrus Extract. Am. J. Clin. Nutr. 48(3): 4

Yeh, C.-C., Kao, S.-J., Lin, C.-C., Wang, S.-D., Liu, C.-J., and Kao, S.-T. (2007). The immunomodulation of endotoxin-induced acute lung injury by hesperidin in vivo and in vitro. Life Sci. 80(20): 1821-1831. 